\title{
High-Bias-Field Operation of GaAs Photoconductive Terahertz Emitters
}

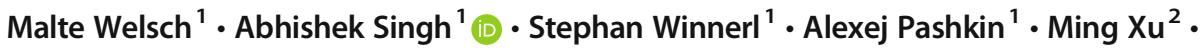 \\ Mengxia $\mathrm{Li}^{2} \cdot$ Manfred Helm ${ }^{1,3} \cdot$ Harald Schneider $^{1}$
}

Received: 4 October 2020 / Accepted: 4 February 2021 / Published online: 5 May 2021

(C) The Author(s) 2021

\begin{abstract}
We demonstrate experimentally the increase of optical-to-terahertz conversion efficiency for GaAs-based photoconductive terahertz emitters. This increase is achieved by preventing device breakdown through series resistors, which act as a current limiter. Pulsed photoexcitation and potential current fluctuations result in heat dissipation leading to local heating, which further increases the current and may lead to device breakdown. We manage to increase the maximum bias field before device breakdown by a factor of 3 under illuminated conditions. For a laser system with $250-\mathrm{kHz}$ repetition rate, the terahertz emission amplitude increases linearly with applied bias field up to $120 \mathrm{kV} / \mathrm{cm}$ bias field, which results in 3 times higher signal as compared to the standard device. Furthermore, we have also achieved this expanded breakdown prevention at $78-\mathrm{MHz}$ repetition rate, where an integrated on-chip resistance leads to an enhancement of the terahertz field amplitude by $70 \%$. This simple technique can increase the performance of almost all photoconductive terahertz emitters by using appropriate resistances according to the emitter capacitance and laser repetition rate.
\end{abstract}

Keywords Terahertz source $\cdot$ photoconductive terahertz emitter $\cdot$ photoconductive antenna

\section{Introduction}

$\mathrm{THz}$ radiation falls in the energy range of many interesting excitations in biological, chemical, and physical processes $[1,2]$. Unfortunately, the generation of significantly intense $\mathrm{THz}$

Abhishek Singh

absingh87@gmail.com; a.singh@hzdr.de

1 Institute of Ion Beam Physics and Materials Research, Helmholtz-Zentrum Dresden-Rossendorf, Bautzner Landstrasse 400, 01328 Dresden, Germany

2 Department of Applied Physics, Xi'an University of Technology, Xi'an 710048, China

3 Institute of Applied Physics, TU Dresden, 01062 Dresden, Germany 
radiation is quite challenging. There are a variety of emission techniques used to get $\mathrm{THz}$ radiation depending on the application of the $\mathrm{THz}$ radiation. Common sources for $\mathrm{THz}$ spectroscopy at room temperature using moderate optical pump energies are $\mathrm{THz}$ emission from photoexcited semiconductors: unbiased devices like surface field emitters and photoDember emitters (vertical and lateral), and biased devices like photoconductive emitters (PCE). The electric field amplitude of THz pulses can be easily controlled by the applied bias on the PCE emitter. PCEs also offer a scattering-free high-frequency electrical chopping for lock-in detection. These properties make PCEs one of the most useful emitters for THz timedomain spectroscopy using mode-locked femtosecond lasers [3, 4].

The basic concept of PCEs is a photo switch, i.e., two biased electrodes on top of a photoconductor. The electron-hole pairs photo-generated by a sub-ps optical pulse in the photoconductor are accelerated by the bias field and emit $\mathrm{THz}$ radiation. The emitted field $E_{\mathrm{THz}}$ is proportional to the time-dependent change of the photocurrent $I_{\mathrm{PC}}$,

$$
E_{\mathrm{THz}} \propto \frac{d}{d t} I_{\mathrm{PC}}
$$

Unfortunately, the optical-to-THz conversion efficiency of PCEs is usually far below $1 \%$. In the last decades, plasmonic light concentrators and plasmonic contact electrodes [5], as well as large-area emitters, have been developed to enhance the emitted signal [6]. Another and much simpler way to enhance the emission efficiency for $\mathrm{THz}$ radiation could be the application of high bias electric fields. However, the current flowing through the PCE emitter generates heat and can cause the thermal breakdown of the emitter, thus restricting the maximum applied bias on the emitter. To solve the thermal breakdown problem of PCE emitters, bias-free PCEs have gained interest in recent times [7, 8]. There have been a few successful attempts to prevent thermal breakdown of the PCEs by using voltage pulses at reduced duty cycles to bias the emitter or by ion implantation of SI-GaAs wafers $[9,10]$. Here, we present concepts that are technologically very simple to prevent breakdown and enhance the emission.

\section{Motivation and Background}

In semi-insulating GaAs, the limitation for the bias field is caused by impact ionization of the EL2 deep donor level (which compensates shallow acceptor levels [11] and thus leads to semiinsulating behavior). Under dark conditions, this breakdown typically sets in at around 100$150 \mathrm{kV} / \mathrm{cm}$. In this field regime, the impurity breakdown usually exhibits metastable behavior [12]. Under laser illumination, the breakdown field is substantially reduced due to the introduced photocurrent and the concomitant heat. In this case, device breakdown, which has been exploited before in photo-induced switching applications [13], already sets in at bias fields of about $40 \mathrm{kV} / \mathrm{cm}$. When this breakdown has occurred, the device (if not irreversibly damaged) enters a lock-on mode of operation, in which the device remains in a low-resistance state until the field is reduced below a sustaining (or lock-on) field, which is typically below 10 $\mathrm{kV} / \mathrm{cm}$ [12]. In this low-resistance state, the current is typically flowing in one or several narrow filaments, which are stable due to localized heating and induced high conductivity along a specific path connecting the two electrodes. These filamentary conduction channels emerge because of the S-shaped I-V characteristics resulting from impurity breakdown [14, 15]. The formation of a current filament will increase the dark current (and hence total current) through the device, which will further increase the device heating, and eventually the device 
will break down. Images of THz PCEs after breakdown are shown in Fig. 1a and 1b. The device shown in Fig. 1a appears to be destroyed by formation of a current filament. For stable operation of the device, it is necessary that the current jump after pulsed excitation drops back to the original dark current level prior to the excitation before any permanent damage occurs.

Even though the electron dc drift velocity in GaAs exhibits negative differential field dependence at electric fields above a few $\mathrm{kV} / \mathrm{cm}$, THz emission induced by sub-100 fs optical pulses is determined by the initial increase of the photocurrent (see Eq. (1)) when electrons still populate the Gamma valley and their drift mobility is large.

After gaining sufficient kinetic energy, electrons are scattered to the side valleys, where the effective mass is enhanced and the drift velocity is reduced. The resulting transient drift velocity overshoot can be simulated by Monte Carlo calculations for different bias fields. We performed the Monte Carlo simulation using a homemade software. The band structure of three valleys $(\Gamma, \mathrm{L}, \mathrm{X})$ in the non-parabolic approximation is employed. The number of charge carriers/particles is kept constant at $\sim 2.5 \times 10^{4}$. The initial optical energy and carrier concentration are set to be $1.3 \times 10^{-17} \mathrm{~J}$ and $1.3 \times 10^{13}$ $\mathrm{cm}^{-3}$, respectively. As shown in Fig. 1c, a maximum average peak velocity of 6-10 $\times$ $10^{7} \mathrm{~cm} / \mathrm{s}$ is predicted, reached at about $500 \mathrm{fs}$ after excitation for $10 \mathrm{kV} / \mathrm{cm}$ and at $<100 \mathrm{fs}$ for $120 \mathrm{kV} / \mathrm{cm}$. At later times, the drift velocity is of the order of $1 \times 10^{7} \mathrm{~cm} / \mathrm{s} \mathrm{as}$ most of the electrons have reached the side valleys $[16,17]$. According to Eq. (1), the emitted $\mathrm{THz}$ field is linearly proportional to the rate of change of current. Thus, $\mathrm{THz}$ field should be linearly proportional to the acceleration of carriers after photoexcitation. We have estimated the initial acceleration of electrons between the 20- and 40-fs time after the photoexcitation. The results are shown in Fig. 1d, which shows a linear increase with the applied bias. Hence, the emitted $\mathrm{THz}$ field will increase significantly at these high fields, as was also verified experimentally $[18,19]$. High-field operation is thus a promising approach for more efficient THz PCEs.

In this paper, we demonstrate high-field operation of $\mathrm{THz}$ emitters by applying series resistances to the emitter in order to suppress device breakdown. In this way, an enhanced efficiency for $\mathrm{THz}$ emission is achieved since the resistance reduces the average current through the device, thus allowing higher bias fields. For further optimization, we also investigate the effect of different locations of the resistors, as well as $\mathrm{THz}$ emission behavior at different pulse repetition rates. Commercial resistors as well as nanostructured on-chip resistors are used.
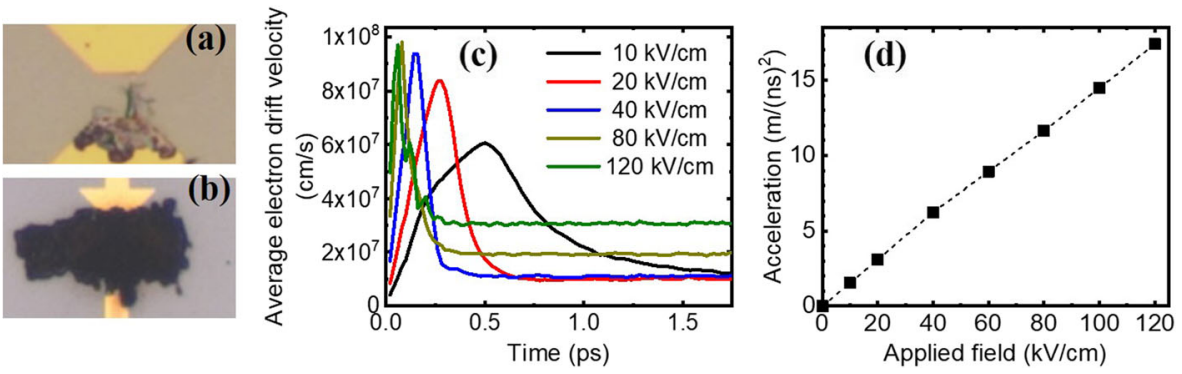

Fig. 1 a, b Microscope images of emitters after device failure. c Average electron drift velocity vs. time in GaAs at varying electric field (Monte Carlo simulation). d The initial acceleration of electrons under varying applied bias field 


\section{Device Configurations, Fabrication, and Setup}

The PCE devices are fabricated by electron-beam lithography. The emitter geometry is a standard bow-tie structure with a 10- $\mu \mathrm{m}$ gap [20]. The electrodes are formed by a 5-nm Ti adhesion layer and a 25-nm Au layer on top of a semi-insulating GaAs substrate. This bow-tie emitter is then equipped with series resistances. The external resistances of $10 \mathrm{k} \Omega$ for each electrode are included in the bias circuit of the emitter in different configurations as shown in Fig. 2a. The distance $x$ between emitter and resistance is varied by inserting a coaxial cable of suitable length between the PCE and the resistor.

The emitter \#A is a standard bow-tie emitter, emitter \#AR $\mathrm{C}_{\mathrm{C}}$ means the resistor is soldered to the copper pads, and emitter $\# \mathrm{AR}_{x}$ means the resistors are located at a distance $x \mathrm{~cm}$ from the emitter. Moreover, another device configuration with on-chip integrated resistors, which will be discussed later, is denoted as \#R (not shown in Fig. 2 a). As shown in Fig. 2b, the equivalent circuit of the emitter can be considered as a capacitor (formed by the device capacitance and cable capacitance) charged through the resistors by the bias voltage supply and discharged via a photo-switch. Inclusion of the resistances in this circuit leads to capacitor charging via resistors, resulting in an increase of the charging time. The coaxial BNC cable between the THz emitter and the resistors also contributes to the capacitance of the system.

Current vs voltage (I-V) characteristics of the emitter without any resistance, and with two resistances of $11 \mathrm{k} \Omega$ each in series (configurations \#A and \#AR , respectively, in Fig. 2a) are studied under dark condition and the data is shown in Fig. 3. A Keithley 2400 Source Meter is used to perform the measurement. The emitter substrate having a semi-insulating nature shows very little dark current of less than $1 \mu \mathrm{A}$ up to the bias of $60 \mathrm{~V}$ and less than $50 \mu \mathrm{A}$ at $120 \mathrm{~V}$ (i.e. $120 \mathrm{kV} / \mathrm{cm}$ bias field). Since the dark resistance of the emitter is several $\mathrm{M} \Omega, 22 \mathrm{k} \Omega$ series resistance does not cause significant change in the dark current. The I-V data shows that we can apply a bias up to $120 \mathrm{~V}$ (i.e., $120 \mathrm{kV} / \mathrm{cm}$ ) on the emitter without destroying it if the photocurrent due to optical pumping of the emitter is small enough to prevent increasing the temperature of the emitter. Assuming that each pump photon at $\sim 800 \mathrm{~nm}$ excites at most one electron-hole pair in the GaAs, and that all excited photocarriers reach the emitter electrode to cause the current flowing through the circuit, a simple calculation shows that $10 \mathrm{~mW}$ pump power can give at maximum $6.4 \mathrm{~mA}$ of photocurrent, which is not expected to cause excessive thermal heating. But, in practice, formation of current filaments by localized heating and/or
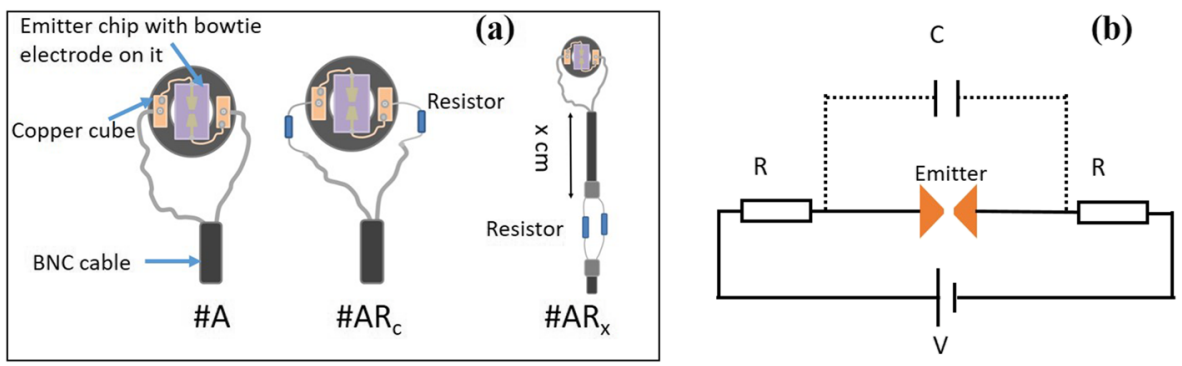

Fig. 2 a Schematic drawing of the different emitter configurations with external resistors. \#A is the standard bow-tie device without series resistors. In $\# \mathrm{AR}_{\mathrm{c}}$, resistors are placed on the copper blocks. \#AR $\mathrm{Ameans}_{x}$ the resistors are placed $x \mathrm{~cm}$ away from the emitter structure and connected by $x \mathrm{~cm}$ long $50 \Omega$ BNC cables. b The equivalent circuit of the emitter with series resistances 
Fig. 3 Dark current through the emitter \#A with and without series resistance. The inset show the same plot for lower voltage range

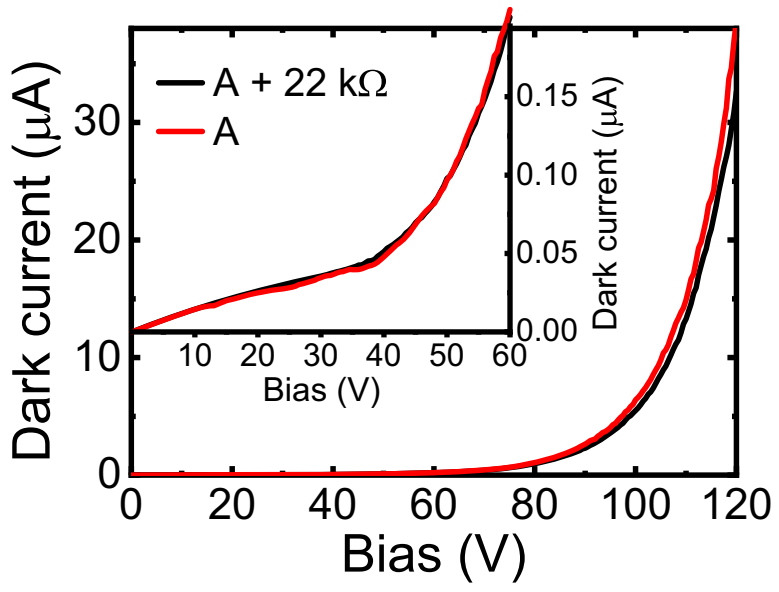

overall device heating takes place at high bias, resulting in very large dark current in the device and subsequent breakdown with permanent damage.

The devices are tested in a standard THz time-domain spectroscopy (TDS) setup using two different Ti:Sapphire laser systems operating at a central wavelength of $800 \mathrm{~nm}$. The first one has 50 -fs pulse width, $250-\mathrm{kHz}$ pulse repletion rate, while the second one has 80 -fs pulse width, $78-\mathrm{MHz}$ repetition rate. A $1-\mathrm{mm}$-thick $\mathrm{ZnTe}<110>$ crystal is used for electro-optic detection.

\section{Results and Discussion}

\subsection{Optical Pumping at 250-kHz Repetition Rate}

First, we discuss the experimental concept and the results obtained with the $250-\mathrm{kHz}$ laser system. Figure $4 \mathrm{a}$ shows the emitted $\mathrm{THz}$ pulses for device configuration $\# \mathrm{AR}_{\mathrm{C}}$ with external resistances of $10 \mathrm{k} \Omega$ adjacent to each electrode. The pump power is $3 \mathrm{~mW}(12 \mathrm{~nJ} /$ pulse), whereas the bias voltage is increased up to $120 \mathrm{~V}$. A standard bowtie emitter \#A was observed to break down already at a bias voltage of 35-45 V. This means that the series resistors prevent device breakdown and increase the maximum $\mathrm{THz}$ field amplitude by almost a factor of 3 . The breakdown field of the device is enhanced by limiting the current through the device. As shown in Fig. 4b, the field amplitude of the emitted $\mathrm{THz}$ pulse exhibits a linear increase with applied bias voltage up to $120 \mathrm{~V}$, which corresponds to an applied field of about $120 \mathrm{kV} / \mathrm{cm}$.

One could naively think that a limited current has to result in reduced $\mathrm{THz}$ emission, but, as shown in Fig. 4c, this is not the case for the chosen operating parameters since the $\mathrm{THz}$ field is radiated within the first picosecond after the carrier excitation by the pump pulse. On this short time scale, the current is not limited by the resistances. The resistances suppress the current through the device just at later times, i.e., after $\mathrm{THz}$ emission is completed, since the antenna structure and the experimental setup result in an effective capacitance which is discharged by the photocurrent pulse. The current responsible for $\mathrm{THz}$ emission has the same value for devices with or without series resistors if the electric bias field across the emitter gap is equal for both configurations. However, if the pump 

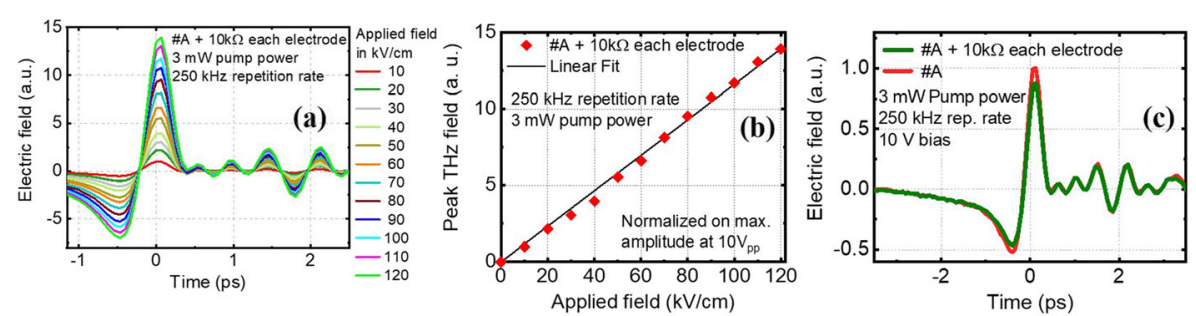

Fig. 4 a Emitted THz pulses from device $\#_{A} R_{C}$ at different bias voltages. b Emitted $\mathrm{THz}$ field vs. bias voltage indicating linear behavior (black line). The series resistors allow for applying high bias voltage and exceeding the breakdown voltage of the device. c A comparison of the $\mathrm{THz}$ emission with and without series resistances

pulse energy is large enough to completely discharge the emitter capacitance $(\sim 0.1 \mathrm{nF}$ including bias cables up to the resistances) and the applied resistance is not small enough to allow complete recharging of the emitter electrode before the arrival of the next pulse (i.e., before $4 \mu$ s for $250-\mathrm{kHz}$ repetition rate pump), the $\mathrm{THz}$ emission will drop. In the present experimental case, although at $3-\mathrm{mW}$ pump pulse energy $\left(\sim 4.8 \times 10^{10}\right.$ photon/ pulse) appears to be large enough to completely discharge the emitter with a capacitance of the order of $0.1 \mathrm{nF}$, which has $\sim 6.2 \times 10^{9}$ electron charges at $10 \mathrm{~V}$, the $R C$ constant of $2 \mu \mathrm{s}$ $(0.1 \mathrm{nF} \times 20 \mathrm{k} \Omega)$ is still smaller than the time period between two pump pulses.

In the following, we discuss the influence of the resistor position on the $\mathrm{THz}$ emission. For this purpose, the external resistors are placed at different distances $x$ away from the emitter (see device \#AR ${ }_{x}$ in Fig. 2). The following distances (i.e., cable lengths) are used: $x=45 \mathrm{~cm}, 135$ $\mathrm{cm}, 180 \mathrm{~cm}, 225 \mathrm{~cm}, 345 \mathrm{~cm}$, and $435 \mathrm{~cm}$. Furthermore, configurations \#A and $\# A R_{C}$ are also included in this study. A low bias voltage of $10 \mathrm{~V}$ is used for this measurement, thus operating in the linear regime without avalanche breakdown. The recorded $\mathrm{THz}$ pulses for the different resistor positions are presented in Fig. 5 a. A clear dependence of the emitted $\mathrm{THz}$ signal strength on the resistor position $x$ is observed.

Device \#A shows the highest THz amplitude of all configurations. The increase of $x$ results in a decrease of the THz signal. This reduction of the THz signal is caused by the increased charging time of the emitter (including the cables up to the resistance). This reduces the actual voltage present at the electrodes at the time of excitation. In Table 1, we list the measured capacitances and corresponding calculated RC time constants for different emitter
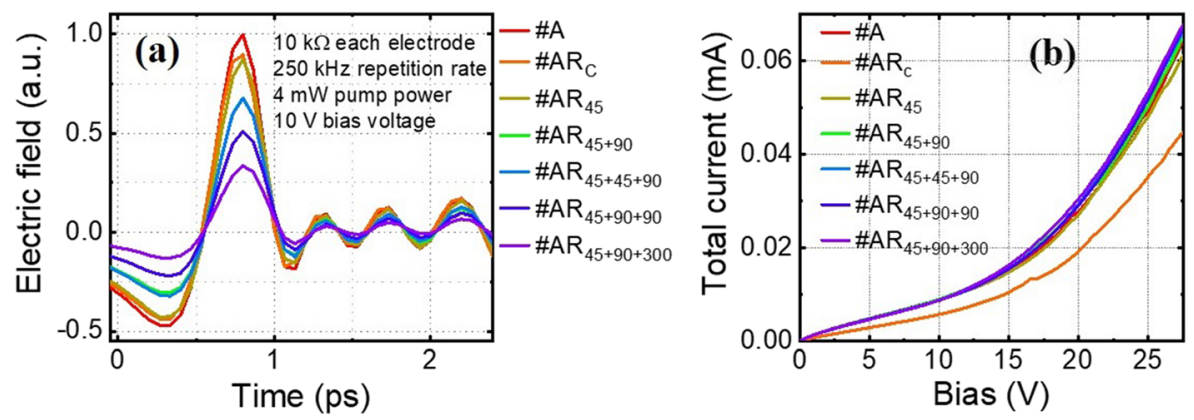

Fig. 5 a THz field transients from PCEs with external resistors placed after BNC cables of different lengths. The $\mathrm{THz}$ amplitude decreases with cable length. b I-V characteristics of the emitters with series resistance at different positions under illumination of 4-mW pump 
Table 1 Measured capacitances, corresponding calculated RC time constants, and $\mathrm{THz}$ emission efficiency for different emitter configurations

\begin{tabular}{lllll}
\hline Emitter configuration & Capacitance $(\mathrm{nF})$ & $\mathrm{RC}$ constant $(\mu \mathrm{s})$ & $\begin{array}{l}\text { Emitter charging } \\
\text { level after } 4 \mu \mathrm{s}\end{array}$ & $\begin{array}{l}\text { Observed THz signal } \\
\text { relative to \#A }\end{array}$ \\
\hline \#A & $<0.097$ & Very small & $100 \%$ & $100 \%$ \\
\#AR $_{\mathrm{c}}$ & $<0.097$ & $<1.94$ & $>87 \%$ & $92 \%$ \\
\#AR $_{45}$ & 0.097 & 1.94 & $87 \%$ & $89 \%$ \\
\#AR $_{45+90}$ & 0.196 & 3.92 & $64 \%$ & $70 \%$ \\
\#AR $_{45+45+90}$ & 0.236 & 4.72 & $57 \%$ & $70 \%$ \\
\#AR $_{45+90+90}$ & 0.269 & 5.38 & $52 \%$ & $52 \%$ \\
\#AR $_{45+90+300}$ & 0.497 & 9.94 & $33 \%$ & $34 \%$ \\
\hline
\end{tabular}

configurations. The capacitance of the emitter including BNC cable up to the resistor position is measured using a multimeter. Operating at $250-\mathrm{kHz}$ repetition rate allows for $4-\mu$ s charging time. Assuming complete discharging of the emitter after each pump pulse, re-charging will occur proportional to the function $(1-\exp (-t / R C))$. With these assumptions, we calculate the charging level of the emitter just before excitation (i.e., charging time of $4 \mu \mathrm{s}$ ) and compare this with the actually observed $\mathrm{THz}$ amplitude relative to emitter configuration \#A (i.e., no resistance and hence $100 \%$ voltage on the electrodes). Although $\mathrm{THz}$ emission depends on resistance position, the $\mathrm{I}-\mathrm{V}$ characteristics shown in Fig. $5 \mathrm{~b}$ do not show any significant change in the total current due to different configurations of the series resistance. The reason is that, for the higher capacitance configurations, the voltage between the electrodes before optical excitation is lower, but due to larger capacitance, the voltage drop across the electrodes is maintained for longer time. Hence, the total current averaged over longer time is almost similar for all emitter configurations.

\subsection{Optical Pumping at 78-MHz Repetition Rate}

According to these results, reducing the capacitance further will allow for high-field operation at much higher repetition rates. To reduce the emitter capacitance after the series resistance, a device \#R with on-chip nanoresistors in series with the emitter is fabricated. A microscope
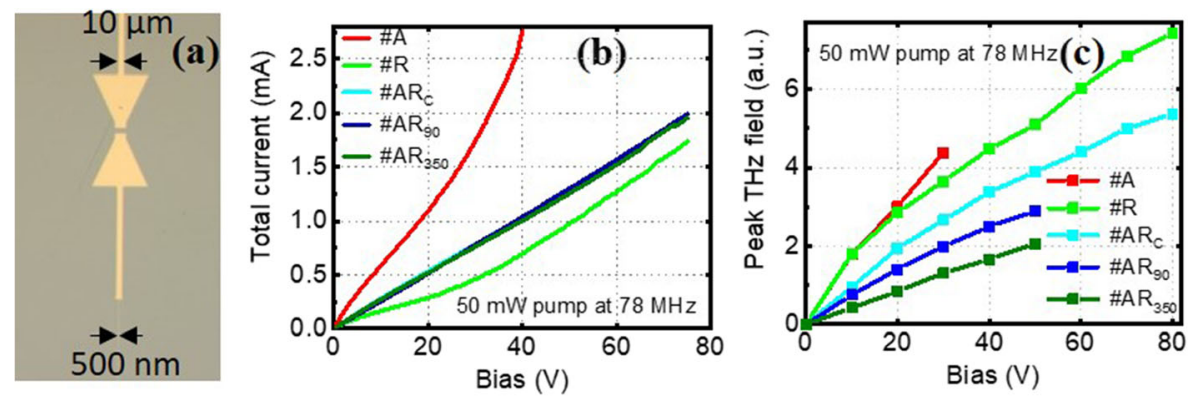

Fig 6 Devices tested at 78-MHz pulse repetition rate. a Microscope image of emitter \#R. Width of the connection line is reduced to $500 \mathrm{~nm}$ for $\sim 3-\mathrm{mm}$ length to achieve the integrated resistance in series. b I-V characteristics of emitters in different configurations under illumination at 50-mW pump power. $\mathbf{c} \mathrm{THz}$ amplitude at increasing bias voltage. External resistances increase the breakdown voltage but the signal strongly decreases. The THz signals from \#R and \#A have almost the same value at the same bias 
image of the emitter \#R is shown in Fig. 6a. The on-chip nanoresistors integrated to the emitter structure are fabricated by narrowing down the connection lines of the emitter from $10 \mu \mathrm{m}$ to $500 \mathrm{~nm}$. The narrowing starts $200 \mu \mathrm{m}$ behind the bowtie structure and is $3500 \mu \mathrm{m}$ long. This distance between the emitter active area and the integrated nanoresistor $(200 \mu \mathrm{m})$ is chosen sufficiently large to ensure that the current is not limited during the emission of the THz pulse. The overall resistance measured using a standard multimeter is around $11 \mathrm{k} \Omega$ for emitter \#A without any series resistance, $32.5 \mathrm{k} \Omega$ for emitter $\# \mathrm{AR}_{x}$ with $11 \mathrm{k} \Omega+11 \mathrm{k} \Omega$ external resistances, and $36.5 \mathrm{k} \Omega$ for emitter \#R with integrated on-chip nanoresistors under the operating conditions of the emitters. We have used the 78-MHz laser to characterize PCE structures with external series resistances $\left(\# \mathrm{AR}_{x}\right)$ and without any series resistance (\#A), as well as the device \#R with the on-chip nanoresistors. I-V characteristics of the emitters under 50-mW illumination are shown in Fig. 6b. The total average current through the emitter \#A increases super-linearly after $20-\mathrm{V}$ bias and it is not advisable to apply higher than $20-\mathrm{V}$ bias on emitter \#A. The emitter \#A breaks down at around 40-V bias. The emitter breakdown is not a reversible process and the actual breakdown bias varies by a few volts from device to device. For emitters \#R and \#AR , the current at higher bias voltages increases linearly with the bias voltage without causing thermal breakdown of the emitter even up to $75 \mathrm{~V}$. This linear behavior of I-V characteristics differs from the I-V characteristics shown in Fig $5 \mathrm{~b}$ at $4 \mathrm{~mW}$ pumping at $250 \mathrm{kHz}$. The reason is that at $50-\mathrm{mW}$ pump power the emitter is getting heated more than in the previous case, and hence, the dark current while operating the emitter is increased (i.e., the emitter resistance is decreased). After reduced emitter resistance, the current is mainly limited by the series resistances, which gives a linear I-V characteristics.

The emitted THz field amplitudes are plotted in Fig. $6 \mathrm{c}$ for a pump power of $50 \mathrm{~mW}(0.64$ $\mathrm{nJ} /$ pulse). As expected, due to the reduced time of $\sim 12 \mathrm{~ns}$ available to charge the emitter electrodes after each excitation pulse, the THz signal is reduced a lot for emitters with external resistances. However, the THz signal from emitter \#R is almost the same as the signal from emitter \#A. This unaffected $\mathrm{THz}$ emission performance of emitter \#R with integrated series resistance is attributed to very small emitter capacitance, which should be of the order of a few $\mathrm{fF}[21,22]$. A reduced $\mathrm{THz}$ signal from other emitter configurations is due to a reduced effective bias on the emitter electrodes at the time of $\mathrm{THz}$ emission (i.e., at the time of photoexcitation). By using on-chip nanoresistors, the breakdown voltage is enhanced and the signal of \#R at $80-\mathrm{V}$ bias is almost 2 times higher than for \#A close to its breakdown. Although low emitter capacitance allows the emitter to quickly recharge, it can also affect $\mathrm{THz}$ emission performance if the capacitance is so low that it cannot maintain the bias on the electrodes even while the $\mathrm{THz}$ emission process lasts. If we compare the electrical energy-to$\mathrm{THz}$ conversion efficiency at $20-\mathrm{V}$ bias, the emitter \#R is almost 3.5 times as efficient as a regular emitter \#A without any resistance.

\section{Conclusion}

The present study demonstrates the advantage of using series resistances for photoconductive $\mathrm{THz}$ emitters. The breakdown of the device can be suppressed, thus enabling device operation at significantly higher bias field. Thus, more efficient $\mathrm{THz}$ emission from the PCE can be achieved. It is necessary to pay attention to the charging times and the capacitances of the emitter structures. In particular, suitable resistances and capacitors have to be chosen. For high-repetition-rate laser oscillators with relatively low pulse 
energy, an on-chip resistor is preferable. When applying low repetition rates, commercial external resistors can be used, whereas the distance between emitter and resistance should be at most a few centimeters. The main result of the present work is the achievement of stable device operation at three times higher bias field, resulting in a threefold increase of the THz field amplitudes. This study demonstrates the possibility of enhancing drastically the optical-to- $\mathrm{THz}$ conversion ratio as well as achieving better signal-to-noise ratio by the simple approach of adding resistors to the emitter circuit.

Acknowledgements The authors thank Mr. Bernd Scheumann for metal deposition for the fabrication of the emitters.

Funding Open Access funding enabled and organized by Projekt DEAL. Support by the Nanofabrication Facilities Rossendorf at IBC is gratefully acknowledged. M. Xu would like to thank the National Natural Science Foundation of China under grant 51877177.

Open Access This article is licensed under a Creative Commons Attribution 4.0 International License, which permits use, sharing, adaptation, distribution and reproduction in any medium or format, as long as you give appropriate credit to the original author(s) and the source, provide a link to the Creative Commons licence, and indicate if changes were made. The images or other third party material in this article are included in the article's Creative Commons licence, unless indicated otherwise in a credit line to the material. If material is not included in the article's Creative Commons licence and your intended use is not permitted by statutory regulation or exceeds the permitted use, you will need to obtain permission directly from the copyright holder. To view a copy of this licence, visit http://creativecommons.org/licenses/by/4.0/.

\section{References}

1. M. Tonouchi, Nat. Photonics 1, 97-105 (2007); https://doi.org/10.1038/nphoton.2007.3

2. H. A. Hafez, X. Chai, A. Ibrahim, S. Mondal, D. Férachou, X Ropagnol, T Ozaki, J. Opt. 18, 093004 (2016), https://doi.org/10.1088/2040-8978/18/9/093004

3. N. M. Burford, M. O. El-Shenawee, Opt. Eng. 56, 010901 (2017); https://doi.org/10.1117/1.OE.56.1.010901

4. C. A. Schmuttenmaer, Chem. Rev. 104, 1759-1779 (2004); https://doi.org/10.1021/cr020685g

5. C. W. Berry, N. Wang, M. R. Hashemi, M. Unlu, M. Jarrahi, Nat. Commun. 4, 1622 (2013); https://doi.org/ $10.1038 /$ ncomms 2638

6. A. Dreyhaupt, S. Winnerl, T. Dekorsy, M. Helm, Appl. Phys. Lett. 86, 121114 (2005); https://doi.org/10. 1063/1.1891304

7. Deniz Turan, Nezih Tolga Yardimci, Mona Jarrahi, 44th International Conference on Infrared, Millimeter, and Terahertz Waves (IRMMW-THz) (2019); https://doi.org/10.1109/IRMMW-THz.2019.8874319

8. M.J. Mohammad-Zamani, M.K. Moravvej-Farshi, M. Neshat, Opt. Express. 23, 19129 (2015); https://doi. org/10.1016/j.ijleo.2019.162975

9. M. Beck, H. Schäfer, G. Klatt, J. Demsar, S. Winnerl, M. Helm, T. Dekorsy, Opt. Express 18(9), 92519257 (2010); https://doi.org/10.1364/OE.18.009251

10. Singh, S. Pal, H. Surdi, S. S. Prabhu, V. Nanal, R. G. Pillay, Appl. Phys. Lett. 2014, 104, 063501; https:// doi.org/10.1063/1.4864623

11. M. Kiyamaa, M. Tatsumi, Appl. Phys. Lett. 86, 012102 (2005); https://doi.org/10.1063/1.1844040

12. L. Partain, D. Day, R. Powell, J. Appl. Phys. 74, 335 (1993); https://doi.org/10.1063/1.354113

13. M. Xu, M.X. Li, X.F. Liu, K. Liu, G.H. Qu, V. Wang, L. Hu, H. Schneider, IEEE Electron Dev. Lett. 40, 1136 (2019); https://doi.org/10.1109/LED.2019.2916427

14. G. Schwarz, C. Lehmann, A. Reimann, E. Scholl, J. Hirschinger, W. Prettl, V. Novak, Semicond. Sci. Technol. 15 593-603 (2000).

15. A. Brandi, M Volcker, W. Prett, Appl. Phys. Lett. 55, 238 (1989); https://doi.org/10.1063/1.101918

16. M. Xu, Ch. Dong, W. Shi, IEEE J. Sel. Top. Quant. 23, 3800706, (2017); https://doi.org/10.1109/JSTQE.2016. 2639822

17. G. M. Wysin, D. L. Smith, A. Redondo, Phys. Rev. B 38, 12514 (1988); https://doi.org/10.1103/PhysRevB. 38.12514

18. A. Leitenstorfer, S. Hunsche, J. Shah, M. C. Nuss, W. H. Knox, Phys. Rev. Lett. 82, 5140 (1999); https:// doi.org/10.1103/PhysRevLett.82.5140 
19. J.-H. Son, Th. B. Norris, J. F. Whitaker, JOSA B 11, 2519-2527 (1994), https://doi.org/10.1364/JOSAB.11.002519

20. S. G. Park, K. H. Jin, M. Yi, J. C. Ye, J. Ahn, K. H. Jeong, ACS Nano 6(3), 2026-2031 (2012). https://doi. org/10.1021/nn204542x

21. W. Sha, J.-K. Rhee, T.B. Norris, W.J. Schaff, IEEE Journal of Quantum Electronics 28, 2445-2455, https:// doi.org/10.1109/3.159551

22. P. U. Jepsen, R. H. Jacobsen, S. R. Keiding, JOSA B 13, 2424-2436 (1996); https://doi.org/10.1364/ JOSAB.13.002424

Publisher's Note Springer Nature remains neutral with regard to jurisdictional claims in published maps and institutional affiliations. 